\title{
Genomic profiling of thymoma using a targeted high-throughput approach
}

Jelena Peric ${ }^{1}$, Natalija Samaradzic ${ }^{2}$, Vesna Skodric Trifunovic ${ }^{2,3}$, Natasa Tosic ${ }^{1}$, Jelena Stojsic ${ }^{4}$, Sonja Pavlovic ${ }^{1}$, Dragana Jovanovic ${ }^{2,3}$

\author{
${ }^{1}$ Institute of Molecular Genetics and Genetic Engineering, University of Belgrade, \\ Belgrade, Serbia \\ ${ }^{2}$ University Hospital of Pulmonology, Clinical Centre of Serbia, Belgrade, Serbia \\ ${ }^{3}$ School of Medicine, University of Belgrade, Belgrade, Serbia \\ ${ }^{4}$ Department of Thoracopulmonary Pathology, Service of Pathology, Clinical Centre \\ of Serbia, Serbia
}

Submitted: 28 November 2019

Accepted: 21 December 2019

Arch Med Sci

DOI: https://doi.org/10.5114/aoms.2020.96537

Copyright $\odot 2020$ Termedia \& Banach

\begin{abstract}
Introduction: Thymomas and thymic carcinoma (TC) are the most common neoplasms localised in the thymus. These diseases are poorly understood, but progress made in next-generation sequencing (NGS) technology has provided novel data on their molecular pathology.

Material and methods: Genomic DNA was isolated from formalin-fixed paraffinembedded tumour tissue. We investigated somatic variants in 35 thymoma patients using amplicon-based TruSeq Amplicon Cancer Panel (TSACP) that covers 48 cancer related genes. We also analysed three samples from healthy individuals by TSACP platform and 32 healthy controls using exome sequencing.

Results: The total number of detected variants was 4447, out of which 2906 were in the coding region (median per patient 83, range: 2-300) and 1541 were in the non-coding area (median per patient 44, range: 0-172). We identified four genes, APC, ATM, ERBB4, and SMAD4, having more than 100 protein-changing variants. Additionally, more than $70 \%$ of the analysed cases harboured protein-changing variants in SMAD4, APC, ATM, PTEN, KDR, and TP53. Moreover, this study revealed 168 recurrent variants, out of which 15 were shown to be pathogenic. Comparison to controls revealed that the variants we reported in this study were somatic thymoma-specific variants. Additionally, we found that the presence of variants in SMAD4 gene predicted shorter overall survival in thymoma patients.

Conclusions: The most frequently mutated genes in thymoma samples analysed in this study belong to the EGFR, ATM, and TP53 signalling pathways, regulating cell cycle check points, gene expression, and apoptosis. The results of our study complement the knowledge of thymoma molecular pathogenesis.
\end{abstract}

Key words: somatic profiling, thymoma, next-generation sequencing (NGS).

\section{Introduction}

Thymomas and thymic carcinoma (TC) are rare thymic epithelial tumours (TETs), mostly localised in the anterior mediastinum [1]. Thymomas are not frequent tumours, having an annual incidence of 0.15 patients per 100,000 person-years $[2,3]$. Here we consider thymo-

\author{
Corresponding author: \\ Jelena Peric PhD \\ Laboratory for Molecular \\ Biomedicine \\ Institute of Molecular \\ Genetics and Genetic \\ Engineering \\ University of Belgrade \\ Vojvode Stepe 444a \\ 11010 Belgrade, P.O. Box 23 \\ Serbia \\ e-mail: \\ jelena.kostic@imgge.bg.ac.rs
}


mas that have been represented as a very indolent form of rare tumours, but with the ability for local and regional invasion. They do not exhibit usual cell atypia, and they are frequently associated with paraneoplastic syndromes, including the most common association - myasthenia gravis [4]. According to WHO classification, thymomas are divided into five subcategories: $A, A B, B 1$, $B 2$, and $B 3$ considering cell morphology, degree of atypia, and differentiation of epithelial cells and lymphocytes [5]. Many attempts have been made to identify target genes for successful therapy of TETs. Still, genomic analysis, with the emphasis on disease rarity, which caused a limited number of studies, has revealed several genes related to this pathology, such as EGFR, HER2, KIT, KRAS, and T53 [6].

Next-generation sequencing (NGS) is a powerful tool for sequencing large numbers of samples, which covers the whole genome, the whole exome, or targets genes of interest, enabling large datasets for various types of conditions, such as cancer. This kind of disease arises from germline mutations and/or somatic mutation accumulation during a lifetime, mostly in a context of specific transcriptome profile and epigenetics factors [7] So far, only a few studies have been published using a high-throughput approach explaining the molecular landscape of thymoma. Here we analysed 35 thymoma patients using targeted NGS methodology by TruSeq Amplicon Cancer Panel (TSACP) consisting of 48 solid tumour-related genes frequently involved in tumourigenesis.

\section{Material and methods}

\section{Patients}

The study was approved by the Ethics Committee of the Clinical Centre of Serbia. The study included a cohort of 35 patients with thymoma. All the diagnoses of thymoma were established using histopathological (PH) tissue sample preparations obtained either by surgery procedure or by fine-needle aspiration biopsy (FNAB), where the surgery was not possible to perform. Thymoma were classified according to World Health Organisation (WHO) and the Masaoka-Koga stage classification. Additionally, 35 healthy control samples were analysed.

\section{DNA isolation and library preparation}

Genomic DNA from 35 thymoma patients was isolated from formalin-fixed, paraffin-embedded (FFPE) tumour tissue and from blood of 35 healthy controls (Qiagen, Valencia, USA). The library preparation wasperformed using $250 \mathrm{ng}$ of genomic DNA, according to the manufacturer's protocol (Illumina Inc., San Diego, CA, USA).

\section{TruSeq Amplicon - Cancer Panel}

TruSeq Amplicon - Cancer Panel, TSACP (Illumina Inc., San Diego, CA, USA) targets mutational hotspots in 48 cancer-related genes ( $A B L 1, A K T 1$, $A L K, A P C, A T M, B R A F, C D H 1, C D K N 2 A, C S F 1 R$, CTNNB1, EGFR, ERBB2, ERBB4, FBXW7, FGFR1, FGFR2, FGFR3, FLT3, GNA11, GNAQ, GNAS, HNF1A, HRAS, IDH1, JAK2, JAK3, KDR, KIT, KRAS, MET, MLH1, MLP, NOTCH1, NPM1, NRAS, PDGFRA, PIK3CA, PTEN, PTPN 11, RB 1, RET,SMAD4,SMARCB 1, SMO, SRC, STK11, TP53, and VHL), TSACP consists of 212 amplicons captured by pairs of oligonucleotides designed to hybridise flanking targeted regions of interest. The TSACP platform that we used was designed to detect exclusively low-frequency somatic variants in a mixed cell population (bellow $5 \%$ ), in the most frequently mutated specific hotspot regions, which were highly specifically targeted in the most common mutated oncogenes associated with solid tumours.

\section{TruSight One Sequencing Panel}

TruSight One Sequencing Panel probes were built to cover the main exons, targeting in total a $12 \mathrm{Mb}$ region size. This panel comprises the exons within 4813 genes. The probes were designed to ensure optimal performance and depth coverage, minimum $20 \times$ and maximum $100 \times$, on $95 \%$ of chosen regions.

\section{Bioinformatics analysis}

A FASTQ file was generated by removing lowquality bases (base quality lower than 30) using quality trimming of 3' ends of non-index reads with low-quality score. This step was performed during the alignment procedure using BWA (Burrows Wheeler Aligner). The alignment process of read sequences to the reference genome hg19 generated BAM (Binary Alignment /Map) file using a BWA-SW aligner (an extended version of - Smith-Waterman, adapted for sequence reads longer than $70 \mathrm{bp}$ ). The final step was a variant calling procedure, which detected single nucleotide polymorphisms (SNPS) and insertions/deletions written in Variant Call Format (VCF). For the generation of a final output file VCF we used GATK (Genome Analysis Toolkit, www.broadinstitute/gatk), a Somatic Variant Caller developed by Illumina. The Illumina-developed Somatic Variant Caller in somatic mode was chosen for variant calling. In order to identify and interpret disease-relevant variants from the Variant Call Format (VCF) we used Illumina VariantStudio. Moreover, for variant annotation we used an additional computational tool: Variant Effect Predictor, VEP [8]. The Integrated Genomics Viewer [9] was used for visual evaluation of the data. For summarising genomic alterations within the on- 
cogenes across the group of patients, we used the OncoPrinter[10] tool. For mutation mapping on a linear protein and its domains we used cBioPortal tool Mutation Mapper [11].

\section{Statistical analyses}

Survival probabilities were estimated using the Kaplan-Mayer method, and differences in the survival distributions were evaluated using logrank test [12]. Statistical analyses were performed using SPSS computer software, version 21.0 (IBM Corp). For all analyses, the $P$ values were twotailed, and $p<0.05$ was considered statistically significant.

\section{Results}

In the present study, we used targeted next-generation sequencing (NGS) to analyse $1.225 \times 10^{6} \mathrm{bp}$ sequences from 35 thymoma patients. The TruSeq Cancer Panel (TSACP) was used for somatic variant detection in specific genomic regions, including 212 amplicons in 48 important cancer-related genes. The average coverage was $145 \times$ per targeted sequences, with 75,786 total reads, with median read depth of $595 \times$. The total number of detected variants in present study were 4447, out of which 2906 in coding region (median per patient 83 with a range: $2-300)$ and 1541 in the non-coding area (median per patient 44 with a range: $0-172$ ) (Figure $1 \mathrm{~A}$ ). For further analysis, we only considered variants that potentially modulate protein structure and function, including nonsense $(\mathrm{N})$, frameshift (F), and missense (M) changes (NFM). In all patients, we identified 1963 potential protein modulating variants with a median per patient of 56, in a range from 2 to 195 variants. Eight patients emerged with the highest number of vari-

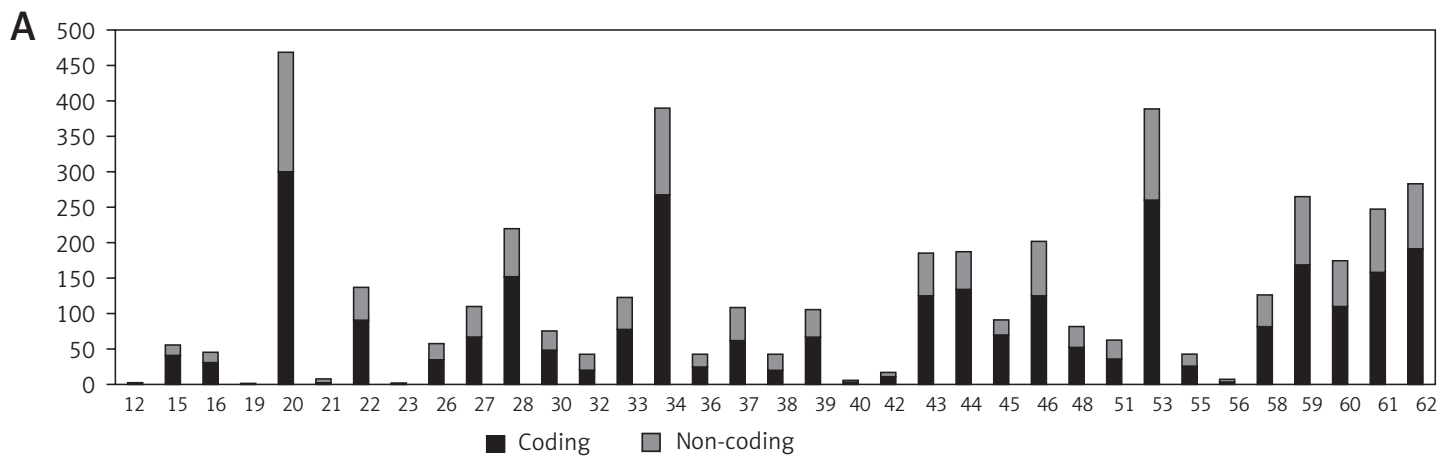

B

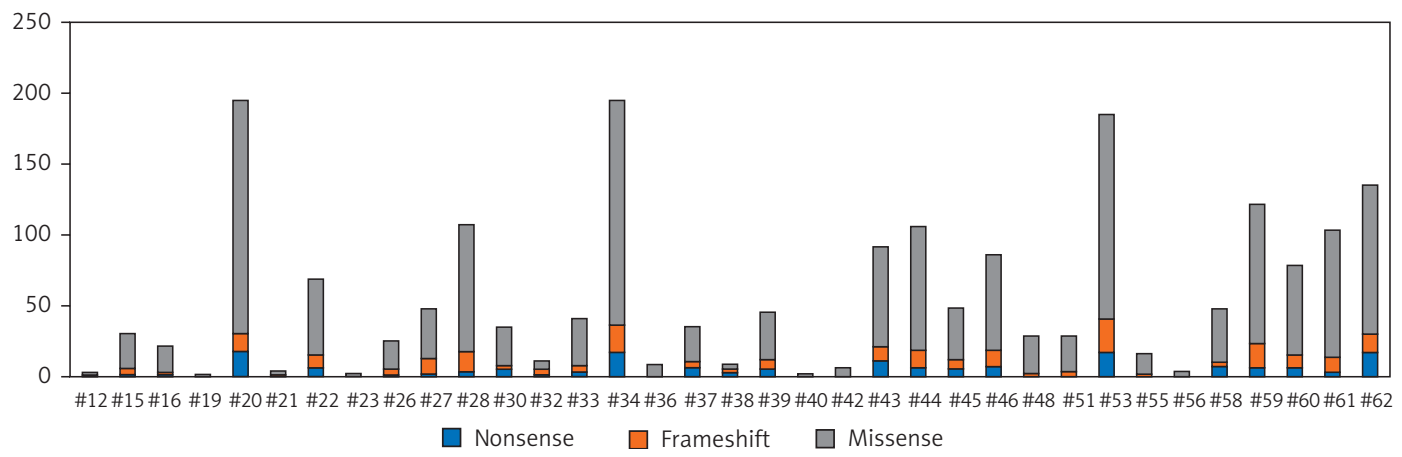

C

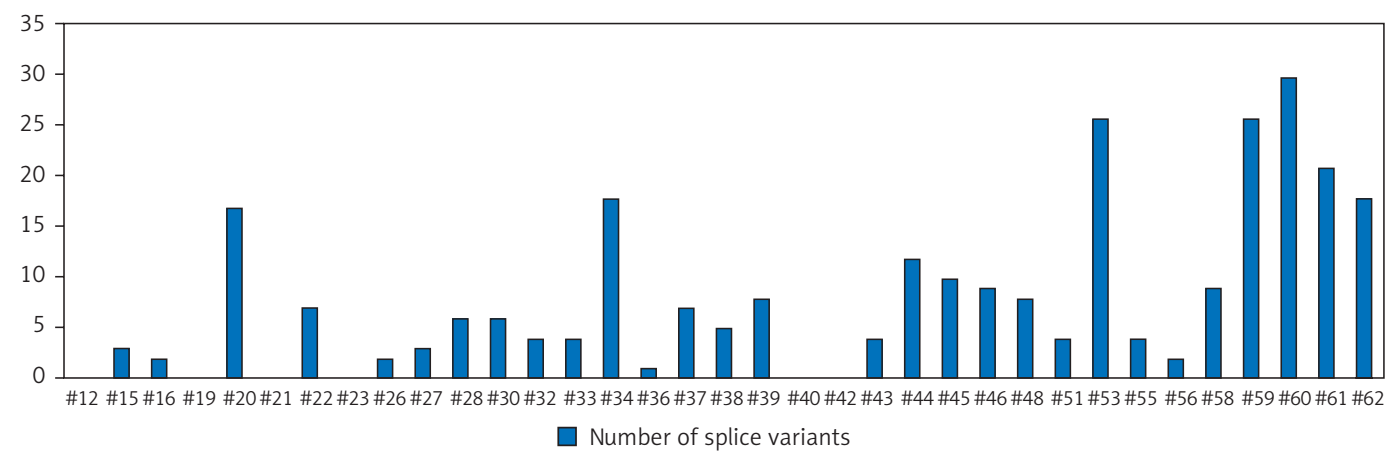

Figure 1. The number of mutations per thymoma patient. A - Total number of mutations in coding and non-coding regions identified by targeted NGS. B - Distribution of nonsense $(N)$, frameshift $(F)$, and missense (M) mutations in coding regions of targeted genes. $\mathbf{C}$ - Distribution of splice variants per patient 


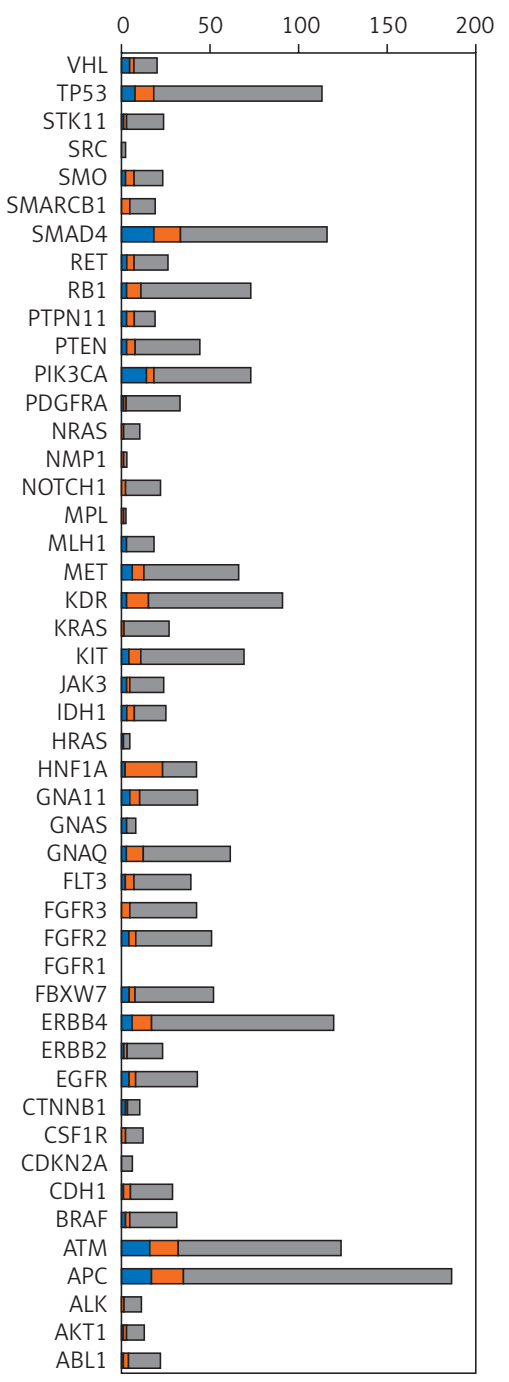

Figure 2. The number of mutations per targeted gene. Distribution of NFM mutations in the coding regions of targeted genes

ants, harbouring more than 100 NFM variants: \#20 (PH tip B3), \#28 (PH tip B1), \#34 (PH tip AB), \#44 (PH tip A), \#53 (PH tip B3), \#59 (PH tip B1), \#61 ( $\mathrm{PH}$ tip $\mathrm{AB}$ ), and \#62 (PH tip $A)$, among them patient \#20 with 195 variants (Figure 1 B). Additionally, we identified 276 splice variants (Figure $1 \mathrm{C}$ ). $\mathrm{PH}$ type $\mathrm{B}$ thymoma was present in $50 \%$ of patients, but observing subtypes, we had almost equal distribution of subtypes among the cases: two patients were detected by each subtype $A$, $A B, B 1$, and $B 3$, while $B 2$ was not present in this group of patients.

Four genes appeared as the highest mutated genes, including $A P C, A T M, E R B B 4$, and SMAD4, having more than 100 NFM variants, indicating a potential contribution of these genes in thymoma pathogenesis. Additionally, EGFR, FBXW7, FGFR3, FGFR2, GNAQ, GNA11, HNF1A, KIT, MET, PIK3CA, PTEN, and RB1 were highly mutated harbouring more than 40 NFM changes. TP53 and

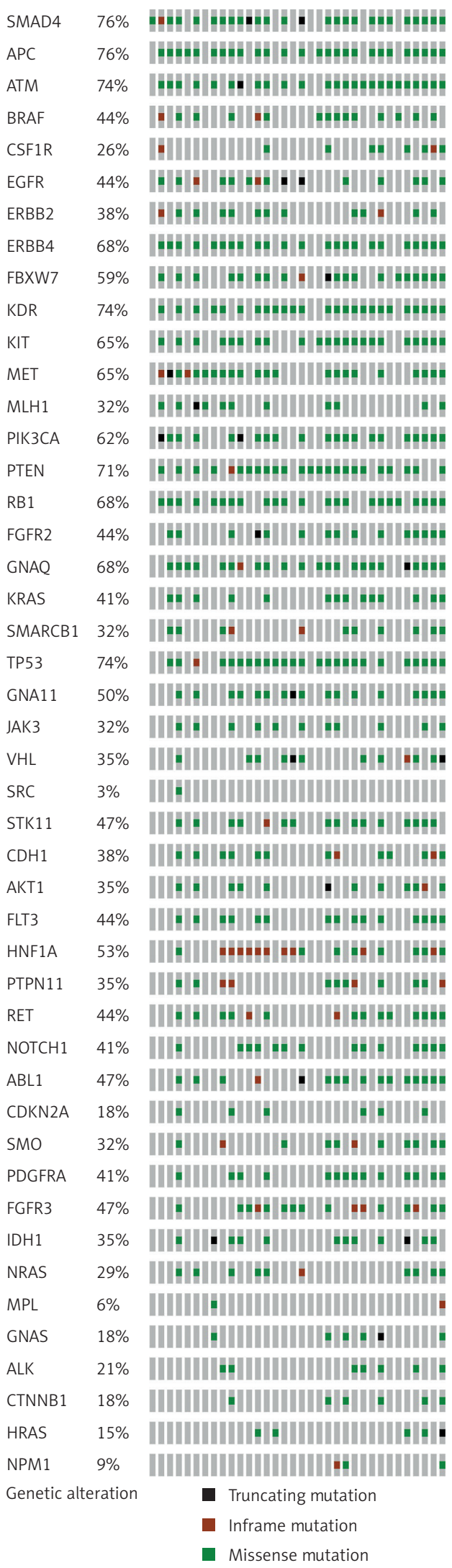

Figure 3. OncoPrint showing the distribution of genetic alterations in 48 targeted tumour suppressor and oncogenes in 35 thymoma patients. The type of mutations are labelled in the colour legend, particular genes in rows, and tumour samples in columns 

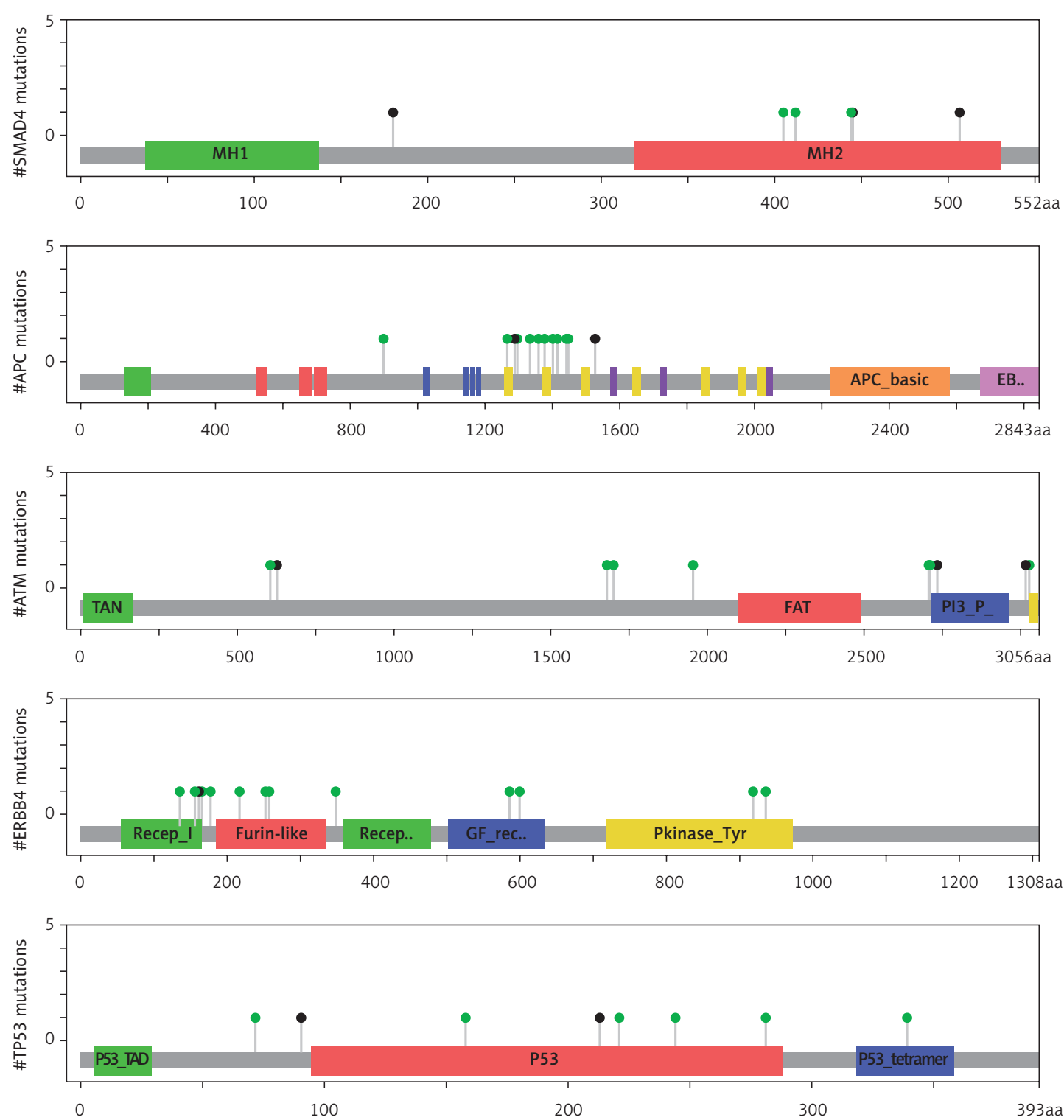

Figure 4. The protein sequence and functional domains are depicted on the $x$-axis. The number of $S M A D 4, A P C$, $A T M, E R B B 4$, and TP53 variants is depicted on the y-axis. Green circles correspond to missense variants. Black circles correspond to nonsense variants

KDR contained more than 90 NFM variants, out of which the majority were well-known polymorphisms (rs1042522 and rs1870377). On the other hand, some of the genes selected using the TSACP panel including FGFR1, MPL, NPM, and SRC had less than five NFM variants (Figure 2).

Protein-changing variants in SMAD4, APC, ATM, PTEN, KDR, and TP53 were present in more than $70 \%$ of analysed cases. The distribution of somatic variant types in 48 selected cancer-related genes across the patients is represented in OncoPrint (Figure 3).

Moreover, this study revealed 168 recurrent variants, which were present at least in two samples in the analysed cohort of patients, out of which 25 were stop gained, nine frameshift, and 134 sub- stitution variants. The recurrent variants within the genes with the highest number of NFM variants are presented with map mutations on linear protein and its domain (lollipop plots) (Figure 4).

For further analysis, we selected previously reported variants with pathogenic effect. Variant pathogenicity was confirmed using SIFT and PolyPhen, and was checked in the dbSNP, COSMIC, OncoKB, ICGC Data Portal, and ClinVar databases. The pathogenic variants and their functional impact are presented in Table I.

Additionally, we analysed three samples from healthy individuals using an amplicon-based TSACP platform, which includes pathogenic somatic variants detected in this study. None of the somatic variants found in thymoma patients 
Table I. Pathogenic variants in thymoma patients

\begin{tabular}{lcccccc}
\hline Sample ID & Gene & Variant position & $\begin{array}{c}\text { Amino acid } \\
\text { change }\end{array}$ & Read depth & $\begin{array}{c}\text { Allele freqencies } \\
\text { in European popu- } \\
\text { lations }\end{array}$ & $\begin{array}{l}\text { Databases } \\
\text { dbSNP } \\
\text { COSMIC }\end{array}$ \\
\hline$\# 20, \# 39$ & SMAD4 & $18: 48581234$ & Q180* & 200 & 0 & rs377767332 \\
\hline$\# 32, \# 59$ & SMAD4 & $18: 48603032$ & R445* & 592 & 0 & rs377767360 \\
\hline$\# 53, \# 60$ & SMAD4 & $18: 48604697$ & K507* & 100 & 0 & COSM1389099 \\
\hline$\# 34, \# 53$ & APC & $5: 112175158$ & C1289* & 473 & 0 & rs1554085355 \\
\hline$\# 34, \# 53$ & APC & $5: 112175876$ & Q1529* & 247 & 0 & COSM1183187 \\
\hline$\# 20, \# 62$ & ATM & $11: 108123623$ & Q628* & 158 & 0 & COSM6067449 \\
\hline$\# 30, \# 53$ & ATM & $11: 108206617$ & Q2733* & 1014 & 0 & COSM6580728 \\
\hline$\# 20, \# 34$ & TP53 & $17: 7577097$ & D281N & 143 & 0 & COSM10943 \\
\hline$\# 20, \# 53$ & TP53 & $17: 7577551$ & G244S & 236 & 0 & COSM10941 \\
\hline$\# 28, \# 34$ & TP53 & $17: 7578458$ & R158C & 294 & 0 & COSM11087 \\
\hline$\# 34, \# 43$ & TP53 & $17: 7578188$ & E221K & 358 & 0 & COSM126982 \\
\hline$\# 30, \# 38$ & TP53 & $17: 7578212$ & R213* & 647 & 0 & COSM10654 \\
\hline$\# 39, \# 58$ & TP53 & $17: 7579415$ & V91* & 113 & 0 & COSM323933 \\
\hline$\# 20, \# 44$ & KRAS & $12: 25398279$ & V14I & 32 & 0 & rs104894365 \\
\hline$\# 20, \# 61$ & RET & $10: 43609949$ & C634Y & 117 & 0 & COSM12722 \\
\hline & & & & & rs75996173 \\
\hline & & & & COSM1237919 \\
\hline
\end{tabular}

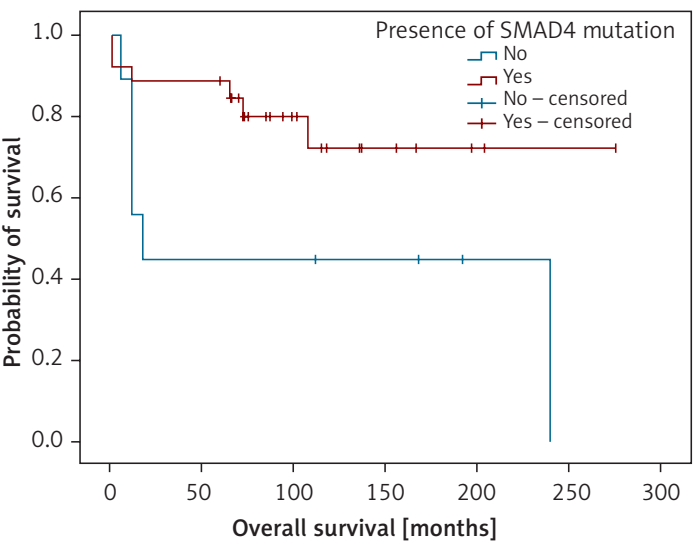

Figure 5. Kaplan-Meier analysis of overall survival in respect to the presence of damaging SMAD4 variants. Patients with SMAD4 mutation $(n=26$; 20 censored), patients not containing SMAD4 mutations ( $n=9 ; 3$ censored). LogRank test $\left(\chi^{2}=4.022 ; p=0.045\right)$, positive vs. negative group -231 vs. 113 months

were detected in controls. Moreover, we analysed 32 healthy control samples using exome sequencing covering pathogenic variants identified in this study (TruSight One Platform, Illumina). Using this approach, which detects germline variants, we did not identify any variant found in thymoma patients. Our findings point out that the variants we reported in this study are somatic thymomaspecific variants.

Also, analysing genetic findings and clinical data we were able to establish that the presence of variants in SMAD4 gene predicted shorter overall survival in the patients (Figure 5 ).

\section{Discussion}

Advances in next-generation sequencing (NGS) of tumour genome have enabled the discovery of novel variants, included single variants (SNVs), small insertions/deletions (indels), copy number variations (CNVs), and translocations, related to tumourigenesis. Identification of genomic alterations using NGS leads to the discovery of new biomarkers in order to improve diagnosis, prognosis, and, finally, treatment of various cancer types [7]. The most comprehensive high-throughput approach, whole-genome sequencing (WGS), enables assessment of all potentially damaging changes in the genome. The disadvantages of this methodology are high cost per sample and low read depth, which limits their potential to detect SNVS with low allele frequencies (less than 5\%), and the time needed for sequencing and data analysis. Consequently, targeted NGS, which targets hotspot regions or specific genes of interest, has been frequently applied. Targeted NGS provides high coverage, which is necessary for detection of rare variants in heterogeneous tumours or in low-purity samples $[13,14]$. Our aim was to study somatic variants in the thymoma genome. We used the TSACP, a previously validated targeted gene panel $[16,17]$. For variant calling we used a somatic caller, which detects somatic variants, 
and the coverage that we obtained is sufficiently high to confirm the presence of somatic variants. The comparison of tumour samples with matched control of non-tumour samples of each patient is very important, in order to eliminate false positive and germline variants. The limitation of our study is that the control non-tumour samples, for example, a buccal swab of each patient, were not available. Instead of this, we included three samples from healthy individuals analysed on the TSACP platform and 32 samples analysed on clinical exome sequencing. Because we did not detect in any control sample variants considered to be either somatic (TSACP) or germline (TSO panel), we presume that the variants identified in this study are somatic thymoma-specific variants.

We found four genes, namely: APC, ATM, $E R B B 4$, and SMAD4, having more than 100 protein-changing variants. Particular variants in these genes were recurrent (appearing in at least two patients). Moreover, EGFR, FBXW7, FGFR3, FGFR2, GNAQ, GNA11, HNF1A, KIT, MET, PIK3CA, $P T E N$, and $R B 1$ genes were highly mutated harbouring more than 40 NFM variants, while TP53 had 90 NFM changes including familiar polymorphism rs1042522. Due to the rarity of the pathology that we investigated, only a few studies concerning high-throughput sequencing datasets related with thymoma have been previously published. Also, the molecular pathology of this disease has been poorly understood, particularly regarding $A, A B, B 1, B 2$, and $B 3$ subtypes. In a previously published study by Belani et al., in which whole genome and whole exome approaches on one cytogenetically normal B3 patient were used, the authors suggested that DNMT3A (p.G728D) and ASXL1 (p.E657fs) variants are involved in thymoma genesis [15]. Petrini et al., using whole genome and transcriptome sequencing, identified one translocation $\mathrm{t}(11 ; \mathrm{X})$, copy number gain of chromosome $1 q, 5,7$ and $X$ and $C N$ loss of $3 p$, 6, 11q42.2-q and q13, 10 SNVs, and two indels, suggesting the need for additional screening for better understanding of disease genetics [16]. The Ekner group analysed two B3 thymoma using an Ion AmpliSeq Cancer Hotspot Panel, and found a mutation in noncoding regions of the SMARCB1 and STK1 1 gene and non-synonymous HRAS mutation in A thymomas [17].

The most frequently mutated genes detected in this study belong to EGFR signalling, ATM, TP53 signalling pathways, regulating cell cycle check points, gene expression, and apoptosis. Analysing the results of the present study, we found that the SMAD4 variants were present in 27 cases. Three of the SMAD4 variants, SMAD4 $\left(\mathrm{Q} 180^{\star}, R 445^{\star}, K 507^{\star}\right)$, were nonsense recurrent variants with probable oncogenic potential according to OncoKB (18), suggesting their potential contribution to thymoma pathogenesis. Moreover, clinical results in our study demonstrated a statistically significant difference in cumulative survival between patients with the presence of damaging variants in SMAD4 and the patients without these variants. Previously published results suggested that pathogenic variants in SMAD4 gene in these tumours were related with poor prognosis of the disease [19]. Some results using a quantitative proteomic approach pointed out SMAD4 as a potential candidate gene with a specific role in the pathogenesis of thymoma [20].

Additionally, we detected pathogenic variants in the following genes: APC (C1289*, Q1529*), ATM (Q628*, Q2733*), TP53 (D281N, G244S, R158C, E221K, R213*, W91*), PTEN (R233Q, KRAS $\mathrm{V} 14 \mathrm{I})$, and RET C634Y, potential targets for drug therapy and drug testing, which could lead a step closer to better treatment and disease outcome. The pathogenic effect of variants was confirmed using SIFT and PolyPhen, and was checked in the dbSNP, COSMIC, OncoKB, ICGC Data Portal, and ClinVar databases.

In this work, we found that the APC gene was mutated in 27 patients. Among the variants that we identified, C1289* and Q1529* emerged as pathogenic variants in this oncogene. APC was characterised as a key tumour suppressor factor involved in several fundamental cellular processes including tumourigenesis and homeostasis, especially of epithelial cells and lymphocytes [21]. In addition to APC, the most frequent genetic variations in all types of thymomas are present in 6p21.3 (MHC locus) and 6q25.2-25.3. But, so-called "common gatekeeper" or common tumour suppressor genes that could be involved in key processes in thymic progenitor cells have not yet been identified. The alterations in APC gene locus have been associated with the aggressive subtypes B2 and B3. "Highrisk alteration" at $A P C$ locus was found in $A B$ type, which are not aggressive forms of the disease, suggesting the existence of another tumour suppressor gene that has the opposite effect to $A P C$ [22].

The ATM gene encodes for the phosphatidylinositol 3-kinase, with the main responsibility of repairing double-stranded DNA breaks. Nextgeneration sequencing analysis pointed out that the ATM gene as one of the most aberrant genes in solid and haematological tumours. The COSMIC database harbours 167 distinct variants related to various tumours, excluding unknown alterations [23]. In our work, ATM was mutated in 26 patients, having two nonsense variants (Q628*, and Q2733*) with a pathogenic effect on the clinical phenotype. 
TP53 has been described previously as the candidate gene related to disease pathogenesis [6]. Non-synonymous mutations in this gene were detected in highly aggressive forms of the disease [17]. TP53 and BCOR were the most frequently mutated genes in TCA and B3 thymomas, respectively [19]. We identified two TP53 variants (R213* and V91*) with stop codon producing truncated protein probably with damaging function, and four pathogenic missense variants (D281N, G244S, R158C, E221K).

Additionally, we found the KDR gene, encoding for tyrosine protein kinase, which had variants in $74 \%$ of cases with one nonsense recurrent variant and eight missense recurrent variants. Moreover, the PTEN gene, which has missense variants with various oncogenic level, were present in $71 \%$ of patients.

The most recent studies on thymoma and other pathologies like glioblastomas and lung adenocarcinoma are related to transriptomic profiling [24-28]. Nevertheless, there amplicon-based technology that we used for thymoma analysis provides reliable and usable data for optimal treatment options, due to its high coverage detection of low-frequency somatic variants. Small-targeted panels with high coverage have already been accepted for translational research, molecular diagnostics, and targeted therapy [29,30]. Our results could contribute to design of thymoma-specific small-targeted panel, applicable in clinical practice. Moreover, cell lines harboring pathogenic variants detected in this study would be a valuable source for drug testing.

In conclusion, a big challenge in precision medicine, imply identification and distinction between rare driver variants, responsible for disease development, and passenger's mutations, which have no impact on tumour phenotype. All somatic variants within the hotspot regions of the observed genes that we identified contribute both to the knowledge of molecular pathogenesis of the thymoma and to the understanding of the clinical phenotypes of the patients. However, further high-throughput screening is needed to complement and improve current knowledge and provide insight into this rare pathology.

\section{Acknowledgements}

Jelena Peric and Natalija Samaradzic contributed equally to this work.

This work was funded by the Ministry of Education, Science, and Technological Development, Republic of Serbia (grant no. III 41004) and by the European Commission, EU-FP7-REGPOT-316088, 2013-2016.

\section{Conflict of interest}

The authors declare no conflict of interest.

\section{References}

1. Cowen D, Richaud P, Mornex F, et al. Thymoma: results of a multicentric retrospective series of 149 non-metastatic irradiated patients and review of the literature. FNCLCC trialists. Federation Nationale des Centres de Lutte Contre le Cancer. Radioth Oncol 1995; 34: 9-16.

2. Engels EA, Pfeiffer RM. Malignant thymoma in the United States: demographic patterns in incidence and associations with subsequent malignancies. Int I Cancer 2003; 105: 546-51.

3. Tomaszek S, Wigle DA, Keshavjee S, Fischer S. Thymomas: review of current clinical practice. Ann Thorac Surg 2009; 87: 1973-80.

4. Riedel RF, Burfeind WR Jr. Thymoma: benign appearance, malignant potential. Oncologist 2006; 11: 887-94.

5. Okumura M, Ohta M, Tateyama H, et al. The World Health Organization histologic classification system reflects the oncologic behavior of thymoma: a clinical study of 273 patients. Cancer 2002; 94: 624-32.

6. Okuda K, Moriyama S, Haneda H, Kawano O, Nakanishi R. Specific mutations in thymic epithelial tumors. Mediastinum 2017; 1. doi: 10.21037/med.2017.10.07.

7. Raphael BJ, Dobson JR, Oesper L, Vandin F. Identifying driver mutations in sequenced cancer genomes: computational approaches to enable precision medicine. Genome Med 2014; 6: 5.

8. McLaren W, Gil L, Hunt SE, et al. The ensembl variant effect predictor. Genome Biol 2016; 17: 122.

9. Robinson JT, Thorvaldsdottir H, Winckler W, et al. Integrative genomics viewer. Nat Biotechnol 2011; 29: 24-6.

10. Gao J, Aksoy BA, Dogrusoz U, et al. Integrative analysis of complex cancer genomics and clinical profiles using the cBioPortal. Sci Signal 2013; 6: pl1.

11. Cerami E, Gao J, Dogrusoz U, et al. The cBio cancer genomics portal: an open platform for exploring multidimensional cancer genomics data. Cancer Discov 2012; 2: 401-4.

12. Bland JM, Altman DG. The logrank test. BMJ 2004; 328: 1073.

13. Duncavage EJ, Abel HJ, Szankasi P, Kelley TW, Pfeifer JD. Targeted next generation sequencing of clinically significant gene mutations and translocations in leukemia. Mod Pathol 2012; 25: 795-804.

14. Xu H, DiCarlo J, Satya RV, Peng Q, Wang Y. Comparison of somatic mutation calling methods in amplicon and whole exome sequence data. BMC Genom 2014; 15: 244.

15. Belani R, Oliveira G, Erikson GA, et al. ASXL1 and DNMT3A mutation in a cytogenetically normal B3 thymoma. Oncogenesis 2014; 3: e111.

16. Petrini I, Rajan A, Pham T, et al. Whole genome and transcriptome sequencing of a B3 thymoma. PLoS One 2013 8: e60572.

17. Enkner F, Pichlhofer B, Zaharie AT, et al. Molecular profiling of thymoma and thymic carcinoma: genetic differences and potential novel therapeutic targets. Pathol Oncol Res 2017; 23: 551-64.

18. Chakravarty D, Gao J, Phillips SM, et al. OncokB: a precision oncology knowledge base. JCO Precis Oncol 2017; 2017. doi: 10.1200/PO.17.00011

19. Moreira AL, Won HH, McMillan R, et al. Massively parallel sequencing identifies recurrent mutations in TP53 in thymic carcinoma associated with poor prognosis. J Thorac Oncol 2015; 10: 373-80.

20. Zhao T, Wu J, Liu X, Zhang L, Chen G, Lu H. Diagnosis of thymic epithelial tumor subtypes by a quantitative proteomic approach. Analyst 2018; 143: 2491-500.

21. Aoki K, Taketo MM. Adenomatous polyposis coli (APC): a multi-functional tumor suppressor gene. J Cell Sci 2007; 120 (Pt 19): 3327-35. 
22. Strobel P, Hohenberger P, Marx A. Thymoma and thymic carcinoma: molecular pathology and targeted therapy. J Thorac Oncol 2010; 5 (10 Suppl 4): S286-90.

23. Choi M, Kipps T, Kurzrock R. ATM mutations in cancer: therapeutic implications. Mol Cancer Ther 2016; 15: 178191.

24. Radovich M, Pickering CR, Felau I, et al. The integrated genomic landscape of thymic epithelial tumors. Cancer Cell 2018; 33: 244-58.e10.

25. Yu L, Ke J, Du X, Yu Z, Gao D. Genetic characterization of thymoma. Sci Rep 2019; 9: 2369.

26. Hou Z, Yang J, Wang H, Liu D, Zhang H. A potential prognostic gene signature for predicting survival for glioblastoma patients. BioMed Res Int 2019; 2019: 9506461.

27. Zuo S, Zhang X, Wang L. A RNA sequencing-based sixgene signature for survival prediction in patients with glioblastoma. Sci Rep 2019; 9: 2615.

28. Wang Y, Zhang Q, Gao Z, et al. A novel 4-gene signature for overall survival prediction in lung adenocarcinoma patients with lymph node metastasis. Cancer Cell Int 2019; 19: 100.

29. Cheng Y, Wang S, Han L, et al. Concurrent somatic mutations in driver genes were significantly correlated with lymph node metastasis and pathological types in solid tumors. Oncotarget 2017; 8: 68746-57.

30. Hirsch B, Endris V, Lassmann S, et al. Multicenter validation of cancer gene panel-based next-generation sequencing for translational research and molecular diagnostics. Virchows Archiv 2018; 472: 557-65. 